\begin{tabular}{|c|c|}
\hline Title & Scattering of light by roughened Gaussian random particles \\
\hline Author(s) & Zubko, Evgenij; Muinonen, Karri; Shkuratov, Y uriy; V ideen, Gorden; Nousiainen, Timo \\
\hline Citation & $\begin{array}{l}\text { Journal of Quantitative Spectroscopy and Radiative T ransfer, 106(1-3), } 604.615 \\
\text { https://doi.org/10.1016/.jpsrt.2007.01.050 }\end{array}$ \\
\hline Issue Date & 2007 \\
\hline Doc URL & http:/hdl.handle.net/2115/28212 \\
\hline Type & article (author version) \\
\hline File Information & JQSR106-1-3.pdf \\
\hline
\end{tabular}

Instructions for use 


\title{
Scattering of light by roughened Gaussian random particles
}

Evgenij Zubko ${ }^{1,2,3}$, Karri Muinonen ${ }^{3}$, Yuriy Shkuratov ${ }^{2}$, Gorden Videen ${ }^{4}$, and Timo Nousiainen $^{5}$

${ }^{1}$ Institute of Low Temperature Science, Hokkaido University Kita-ku North 19 West 8, Sapporo 0600819, Japan

${ }^{2}$ Astronomical Institute of Kharkov National University, 35 Sumskaya St., Kharkov, 61022, Ukraine ${ }^{3}$ Observatory, P.O. Box 14, FIN-00014 University of Helsinki, Finland

${ }^{4}$ Army Research Laboratory AMSRD-ARL-CI-EM, 2800 Powder Mill Road, Adelphi, Maryland 20783, USA

${ }^{5}$ Department of Physical Sciences, Division of Atmospheric Sciences, P.O. Box 64, FI-00014 University of Helsinki, Finland

\begin{abstract}
.
We study the influence of surface roughness on light scattering by Gaussian-random-rough particles. Altogether, we consider four kinds of roughened surfaces: small-scale or large-scale roughening of the particle surface layers that are either thin or thick. We have performed computations for two realistic values of refractive indices corresponding to water ice $(m=$ $1.313+0 i)$ and silicates $(m=1.6+0.0005 i)$. We vary the circumscribing-sphere size parameter $x_{c s}$ from 2 up to 12 in the case of silicate particles and up to 14 in the case of icy particles. For all sets of parameters, scattering of light by roughened particles is noticeably different from scattering by unroughened Gaussian particles. The parameter most sensitive to roughening is the degree of linear polarization of the scattered light when illuminated by unpolarized incident light. Except for the backscattering regime, roughening of particles increases the degree of polarization. Near backscattering, the dependence is more complicated. In the case of icy particles, roughening typically decreases the amplitude of the negative polarization surge; whereas, in the case of silicate particles, the dependence is non-monotonic: for $x_{C S}<10$, roughening neutralizes the surge; whereas, for $x_{C S}=10$ and 12, it makes the surge more pronounced. Particles with large spatial scale roughness produce less negative polarization than those with small-scale roughness. Particles with thin roughened surface layers generally produce larger negative polarization surges than those with thick layers. The intensity of scattered light is sensitive to roughening mostly near the backscattering although, for silicate particles with thick layers of roughened surface, we detect its influence also near forward scattering. In the case of icy particles for $x_{c s}=12$ and 14, thick-layer roughness
\end{abstract}


amplifies the intensity of backscattering; whereas, for other cases, roughening results in decreased backscattering. Particles with thick roughened layers produce typically more gentle angular profiles of intensity near backscattering than the unroughened particles or particles with thin roughened layers. 


\section{Introduction}

Scattering of light by single small particles comparable to the incident wavelength plays an important role in various scientific and technical applications. In some fields of research, for example, in the physics of comets, the sunlight scattered by dust particles is practically the sole source of information about the comets due to the obvious difficulties (and cost) of in-situ sampling. Of course, remote sensing should be based on reliable knowledge of the principles of light scattering by particles. We accrue such knowledge typically through either theoretical or experimental approaches. However in the case of light scattering by dust particles comparable to wavelength both approaches are doomed to fail. The theoretical approach cannot be followed through due to the complexity of the scatterers - the dust particles have random irregular shapes and, most probably, intricate internal structures. As a consequence, no existing analytical solution can be applied directly to describe the scattering by dust particles. By the same token, the extension of conclusions made in the case of spherical particles (when analytical solutions are actually available) to particles of irregular shape does not appear to be justified. Experimental studies of light scattering by particles comparable to wavelength face the challenges in dealing with micron-sized particles. In practice, it implies a lot of difficulties concerning the preparation of sample particles with desired physical properties. Increasing the wavelength of the incident wave allows one to avoid these kinds of problems. For example, Gustafson and Kolokolova [1] used microwaves to study scattering by particles comparable to the wavelength. In such a case, the size of the sample particles is about ten centimeters, removing many of the difficulties described above for micron-sized particles. However, there is the challenge of creating accurate analogues of real particles.

The progress in computer technology during the last twenty years has opened a new approach to study scattering of light by small particles, namely, numerical "experiments." In this approach, a sample particle is generated in computer memory. Then, for given incident wave, the field induced inside the particle is computed. Finally, the scattered wave in the far zone is computed. At the present time, there are 
three well-developed approaches for numerical simulation of scattering of light by particles comparable to the wavelength. These include the discrete-dipole approximation (DDA) [2-8], the $T$-matrix method [9, 10], and the finite-difference time-domain (FDTD) method [11, 12].

In the current paper, we present the results of our study of the surface roughness influence on scattering of light by irregular particles comparable to wavelength. Numerical simulations are appropriate for this study, because we can control the roughening process completely. For the computation of light scattering we use our own well-tested DDA code [6-8, 13]. As initial particles we choose the Gaussian random spheres (GRS) [14]. They are parameterized by the relative radius standard deviation $\sigma$ and power law index in the covariance function of the logarithmic radius $v$. We use a fixed set of 100 sample particles that is generated with $\sigma=0.245$ and $v=4$. A few sample particles are shown in Fig. 1. As one can see, the given set of parameters provides strongly nonspherical particle shapes. At the same time, the particles surfaces are still smooth that is the necessary condition for the study of the roughening of particles.

Although the roughened particles arise in different applications, there are only a few studies of the effect of surface roughness on light scattering. We discuss briefly the most recent investigations of light scattering by roughened particles. Scattering of light by roughened spheres has been carried out in [15] with the help of the FDTD method. Rough spheres are generated via random fluctuation of the radii. The size parameter of the sphere $x(x=2 \pi r / \lambda$, where $r$ is the radius and $\lambda$ is the wavelength) varies from 5 to 20 . Results of this work could be summarized as follows. In the case of a sphere with small-scale roughness at $x=5$, the dependence of the $S_{11}$ element of the scattering matrix on the scattering angle is similar to that of the unroughened sphere, while there are differences in other elements. Increasing the size parameter and/or scale of roughness leads to differences in all elements of the scattering matrix. The angular dependence of linear polarization degree $P\left(P=-S_{21} / S_{11} \cdot 100 \%\right)$ does not show a monotonic dependence on the scale of the roughness. 
In $[16,17]$, the effect of roughness on light scattering by Gaussian random spheres (GRS) has been studied. Particles of this type are formed by superposition of orthonormal spherical harmonics $Y_{l m}$ [14]. The expansion of GRS in series of spherical harmonics is, in principle, infinite, but making use of covariance functions pertaining to single degrees in the Legendre polynomial expansion results in harmonic shapes described by finite series of spherical harmonics corresponding to the degree specified by the covariance function. Therefore, GRS itself allows a systematic study of surface-roughness effects. In [16], computations were carried out using the FDTD algorithm. In this preliminary study, the evolution of the Mueller matrix elements was monitored as the particle shape became extreme. In [17], computations were carried out with the DDA. The value of the mean-radius size parameter was varied from 1 to 9 . Results of this work could be summarized as follows. It is difficult to find truly systematic effects of roughness on scattering. There is no obvious limiting scale of roughness below which light is insensitive to roughness. At $x=1$, light scattering is insensitive to all scales of roughness; whereas, when $x$ is increased, it fast becomes sensitive to all scales of roughness.

To increase the resolution of the particle description and add extremely sharp surface roughness, FDTD was performed on two-dimensional columns [18]. It was noted that roughness smooths the elements of the Mueller matrix and the effects are more pronounced as particle size increases. In [19], high-order Chebyshev particles are analyzed as a means of adding low levels of surface roughness. The authors also examine Mueller matrix elements and largely confirm the results for threedimensional particles.

Note that the roughening algorithm used in [16] affects not only the particle surface but also its shape. Within the present work we propose an alternative complementary algorithm for roughening the surface of an irregular particle. Our algorithm affects only the surface layer of the particle conserving its initial overall shape. The paper is organized in the following way: in Sect. 2, we describe briefly the DDA and our code; in Sect. 3 the algorithm for roughening the particle surfaces; in 
Sect. 4, we show and discuss the results of our simulation; and in Sect. 5, we summarize the conclusions and future prospects.

\section{Scattering computations with the Discrete-Dipole Approximation}

DDA is a powerful approach to compute scattering of light by wavelengthscale particles of arbitrary structure and shape. The main idea of DDA is the representation of a scattering particle by an array of dipoles located in a regular cubic lattice. Via such a dipole representation of the particle, the volume integral equation for the internal electric fields is transformed into a system of linear equations. The unknowns of the system are the polarization vectors induced at each dipole, while the matrix of the free coefficients describes the dipole-dipole interactions (each element of the matrix is a complex-valued $3 \times 3$ matrix). The scattering properties of the target particle can be obtained from the solution of the given system of linear equations.

Although the idea of DDA can be easily understood, it was used for the first time only in 1973 [2] due to computational difficulties. After the first DDA computations, it remained almost unused for a long time. Increasing interest in DDA coincided with the rapid evolution of computer performance in the end of the 1980s. At the same time, new ways were found to optimize DDA. The most important finding concerned the computation of the matrix-vector product. This operation must be carried out for not less than one time per iteration and requires $9 N^{2}$ multiplications of complex values, where $N$ is the number of dipoles. In [3], the authors proposed to reduce the matrix-vector product to a three-dimensional convolution which can be computed by using the fast Fourier transformation (FFT). The FFT allows the computation of the matrix-vector product by only $9 N \log N$ multiplications of complex values. As a consequence, the treatment of larger numbers of dipoles became possible. At present, scatterers composed of hundreds of thousands of dipoles are usually being considered in a straightforward way.

Another advantage of DDA concerns the computation of the dipole polarizabilities from the given value of refractive index of the target material. The well-known relationship of Clausius-Mossotti used in [2] has certain restrictions 
because it has been derived by assuming that the dipole size parameters $x_{\text {dip }}$ $\left(x_{\mathrm{dip}}=2 \pi r_{\mathrm{dip}} \lambda\right.$, where $r_{\mathrm{dip}}$ is the dipole radius and $\lambda$ is the wavelength) equals 0 . Although this relationship works for nonzero sizes of dipoles, the range of its applicability is rather small. Increasing $x_{\text {dip }}$ even to 0.1 leads already to noticeable errors in the light-scattering simulations. The most significant progress in deriving the relationship between the refractive index and dipole polarizability for dipoles with nonzero size parameters took place in [4]. The formula derived was termed the Lattice Dispersion Relation (LDR). As it has been shown in the same manuscript, when the condition $2 x_{\text {dip }}|m| \leq 0.6$ (here $m$ is the refractive index) is fulfilled, LDR provides good agreement of DDA and Mie theory in the case of absorption and extinction cross sections.

To compute light scattering by Gaussian random particles comparable to wavelength we used the well-tested DDA code developed by E. Zubko and Yu. Shkuratov $[6-8,13]$. The code has been written in the $\mathrm{C}++$ programming language and includes the abovementioned improvements of DDA. Additionally, an improved technique of averaging over particle orientations is applied. We divide the process of averaging the light-scattering properties over orientations into averaging over directions of the incident field and averaging over scattering planes for the fixed direction of the incident field. The division is reasonable because the most timeconsuming part of the DDA computation, the computation of the field induced on each dipole, depends on the orientations of the wave vector and polarization vector of the incident field, but not on the observing conditions. The rest of the DDA computations concern the determination of the scattered field in the far zone which can be performed in several different scattering planes simultaneously. Thus, we have the possibility to average over scattering planes with minimum additional consumption of processor time. The number of scattering planes is an input parameter for the code; within the present work, we used 100 scattering planes, which were uniformly distributed around the wave vector of the incident field. It allowed us to obtain statistically reliable results considerably faster in comparison to the common approaches using a single scattering plane. A comparison of the DDA codes 
developed by different programmers has been carried out recently and confirmed the accuracy of our algorithm [13].

The use of a numerical approach to compute light scattering forces us to control the accuracy of our computations. We use a two-level control procedure. On the first level, we test whether the DDA results satisfy Maxwell's equations. This is easy to do in the case of regular particles (spheres, spheroids, clusters of spheres, etc.) by a simple comparison of the DDA solution to the corresponding analytical result. In the case of irregular particles, the problem is more difficult but there are ways to solve it. We compare the values of the scattering cross section $C_{\text {sca }}$ computed in two different ways. One of them is based on the optical theorem [20], which is used to calculate the cross sections for extinction $C_{\text {ext }}$ and absorption $C_{\text {abs }}$. The extinction cross section $C_{\text {ext }}$ results from the mutual interference of the incident field and the field induced on the dipoles; whereas, absorption cross section $C_{\text {abs }}$ results only from the fields induced on the dipoles. Once $C_{\text {ext }}$ and $C_{\text {abs }}$ have been found, the scattering cross section $C_{\text {sca }}$ can be easily computed as $C_{\text {sca }}=C_{\text {ext }}-C_{\text {abs. }}$. Simultaneously, we can compute the scattering cross section $C_{\text {sca }}$ by integrating the intensity of the scattered field over a sphere circumscribing the particle. In this way, $C_{\text {sca }}$ results from the mutual interference of waves scattered by all dipoles to the observation point. From a pure mathematical point of view, the two ways to compute $C_{\text {sca }}$ differ from one another and, in general, there are no reasons to expect that they would give exactly the same result. But if the DDA solution satisfies Maxwell's equations, the values of $C_{\text {sca }}$ obtained in the two ways must be equal. In our present computations, typical differences between the two values of $C_{\text {sca }}$ are about $1-2 \%$.

We average the light-scattering properties of irregular particles over orientation and sample particles by using a Monte-Carlo technique. Although the DDA results satisfy Maxwell's equations, we have additionally to control the statistical reliability of the averaging process. With this aim, we make use of the following procedure. As mentioned before, we perform DDA computations in 100 scattering planes for each orientation of the sample particle. These scattering planes are uniformly distributed around the wave vector of the incident field and we average the light-scattering 
properties over them. Additionally, we perform two subprocesses of averaging; each of them is based on half the number of scattering planes (i.e., 50 scattering planes). We compare the results of both subprocesses to the results of averaging over 100 planes. As a quantitative indicator of the quality of averaging, we use the standard deviation of the linear polarization degree obtained with 100 and 50 scattering planes. Of course, this parameter varies with the scattering angle and, in the current work, we set an upper limit for its fluctuation at $1.5 \%$. We continue the averaging process until the standard deviation of polarization is smaller than this value.

\section{Roughening the surfaces of Gaussian random spheres}

In order to roughen the surfaces of sample particles, we have developed an algorithm based on the DDA representation of the initial particle with an array of dipoles. We divide the dipoles representing a given particle into two classes: those belonging to the surface layer and those belonging to the interior of the particle. To classify a given dipole, we test the nearest neighbors of that dipole. Because the dipoles are placed in a cubical cell, the total number of nearest-neighbor dipole sites equals 26. Thus, if a given dipole has less than 26 neighboring dipoles, it is marked as a surface dipole-otherwise it is an interior dipole. Above, we have described the case where the depth of the surface layer equals 1 dipole, but the thickness can be increased easily. The thickness of the surface layer is a free parameter of our model. In this paper, we examine two thicknesses for the surface layer of particles, namely 1 and 5 dipole diameters.

In what follows, we operate only with the dipoles of the surface layer, while the sub-array of internal dipoles is not modified. Among the surface dipoles, we randomly choose a certain number of sites. Half of them are marked as seed sites for material; whereas, the rest are taken to be seed sites for free space. Each of the remaining surface dipoles receives the same classification as the nearest seed site. If a given surface dipole site has simultaneously nearest seed cells of different properties, it is taken to be a material site. As one can see, the algorithm operates only with three parameters - the thickness of the surface layer, and the numbers of seed cells for 
material and free space. Varying these parameters provides us with different kinds of surface roughness. For each value of surface layer thickness, we tested two sets of seed cells. The first set consists of 1500 seed cells for material and 1500 for free space, and provides small-scale surface roughness on the particles. The second set consists of 150 seed cells for material and 150 for free space, giving large-scale surface roughness.

Images of certain sample particles with various types of surface are shown in Fig. 2. On the left, the initial unroughened particle is shown. Following column of two images presents the cuts of this unroughened particle at two values of the surface layer thickness. The upper and low images correspond to the thickness of 1 and 5 dipole diameters, respectively. Dipoles belonging to the surface layer are marked by white and internal dipoles correspond to dark grey color. The next column of pictures presents the cuts of sample particles with small-scale surface roughness. Finally, the rightmost images show the cut of particles with large-scale surface roughness.

Note that the roughening algorithm decreases the amount of material. While the unroughened Gaussian random sphere is composed, on average, of 19,133 dipoles, roughened particles with small-scale and large-scale roughness in the 1dipole-diameter-thick surface layer consist of 17,032 and 16,565 dipoles, respectively. When the depth of the surface layer equals 5 dipole diameters, particles with small-scale and large-scale roughnesses consist of 13,551 and 12,229 dipoles, respectively.

\section{Discussion of results from simulations}

We calculate the scattering of light at two realistic values of refractive index $m$ in the visible band. These are $m=1.313+0 i$, which corresponds to pure ice, and $m=1.6+0.0005 i$, which corresponds to silicates. Another important parameter of light scattering by particles comparable to the wavelength is the ratio of particle size to wavelength. It is common to describe this ratio with the so-called size parameter $x=2 \pi r / \lambda$, where $r$ is the radius of the particle and $\lambda$ is the wavelength. Because the radius of the particle is not unambiguous in the case of irregular particles, the size 
parameter needs to be defined each time irregular particles are studied. We associate $r$ with the radius of the circumscribing sphere of the largest sample particle of the complete set of unroughened particles. We denote the size parameter by $x_{\mathrm{cs}}$. We note that our algorithm for roughening the particle surface does not significantly change the size of the particles (see Fig. 2) and, thus, the radius of the circumscribing sphere of the largest sample particle has the same value for all sets: unroughened particles and particles with small-scale and large-scale roughness. We determine $r$ with the help of the unroughened particles just for convenience. The value of $x_{\mathrm{cs}}$ has been varied from 2 up to 12 in steps of 2 for silicate particles and up to 14 for icy particles. For a fixed wavelength of $\lambda=0.5 \mu \mathrm{m}$, the resulting size parameters correspond to particle sizes varying from $0.3 \mu \mathrm{m}$ to $1.9 \mu \mathrm{m}$ for silicate particles and $0.3 \mu \mathrm{m}$ to 2.2 $\mu \mathrm{m}$ for ice particles.

We already mentioned in the previous section that our algorithm for roughening the particle decreases the number of dipoles representing the scatterer. Because we study purely the effect of roughness on scattering of light, we correct the size parameter of roughened particles so that the unroughened and roughened scatterers would have identical volumes. When the thickness of the surface layer is equal to 1 dipole diameter, the relationships between the size parameters of initial and roughened particles are as follows:

$$
\begin{aligned}
& x_{\mathrm{cs}}^{\text {small-scale }}=1.039541 \cdot x_{\mathrm{cs}}, \\
& x_{\mathrm{cS}}^{\text {large-scale }}=1.049204 \cdot x_{\mathrm{cs}} .
\end{aligned}
$$

In the case of roughened particles generated with surface layer thickness equal to 5 dipole diameters, the corresponding relationships are

$$
\begin{aligned}
& x_{\mathrm{cs}}^{\text {small-scale }}=1.121865 \cdot x_{\mathrm{cs}}, \\
& x_{\mathrm{cs}}^{\text {large-scale }}=1.160892 \cdot x_{\mathrm{cs}} .
\end{aligned}
$$


Another popular definition of the size parameter is related to the sphere of equivalent volume of material. In this case, $r$ means simply the radius of the sphere of the same volume of a material as the irregular particle. The relationship of this size parameter $x_{\text {eqs }}$ to that of the initial unroughened particles is

$$
x_{\mathrm{eqs}}=0.518351 \cdot x_{\mathrm{cs}} .
$$

Figures 3 and 4 present the results of the simulations of light scattering by unroughened and roughened Gaussian random spheres for $m=1.313+0 i$. Figure 3 shows maps of the intensity (on the left; $\ln I$ ), degree of linear polarization $P$ (in the middle) and the scattering-matrix-element ratio $D=\left(1-\mathrm{S}_{22} / \mathrm{S}_{11}\right) \cdot 100 \%$ (on the right). Although $D$ cannot be observed directly, it contains information about the depolarization characteristics of scatterers. The abscissa on the maps is the scattering angle, whereas the ordinate is the size parameter. Thus, horizontal lines on the maps represent angular dependences and vertical lines size dependences.

The first row of the maps in Fig. 3 corresponds to initial unroughened particles [21]. The second and third rows show results for particles having thin layers of surface roughness (its thickness is equal to 1 dipole diameter). The second row corresponds to the case of small-scale surface roughness while the third corresponds to the case of large-scale surface roughness. Although the maps have low resolution of parameters, they provide an overall view of the influence of roughening on light scattering. As expected, roughening constrained to thin surface layers leads to small changes. Nevertheless, these changes are visible, especially on the maps of intensity and polarization; whereas, the map of $D$ is rather insensitive to roughening of thin layers. We would also like to note that maps of both kinds of roughened particles are similar to one another.

Among the angular scattering patterns, the degree of linear polarization is most sensitive to roughening constrained to the thin surface layer. As one can see from 
Fig. 3, it shows at least three angular regimes that are sensitive to roughening. First, near forward scattering, polarization is increased due to roughening. Second, near the maximum of the positive polarization, roughening again leads to an increase of polarization. Third, for the negative polarization branch near backscattering, we can again establish a tendency of increasing polarization. Influence of shallow roughening of the particle surface on the intensity can be distinguished only in the backscattering region: the intensity decreases due to roughening.

Because the intensity and polarization in the backscattering region are sensitive to roughening, in what follows, we concentrate on these two angular patterns in the backscattering regime. Figure 4 shows the curves of normalized intensity and degree of linear polarization for large scattering angles. The figure consists of four panels with results for $x_{\mathrm{cs}}=8,10,12$ and 14 . Everywhere, the upper screen shows angular dependencies of intensity while the lower screen shows angular dependencies of polarization. The solid line corresponds to that of the initial unroughened particles, the thin short-dashed line to particles with small-scale thin-layer roughness, and the thin long-dashed line to particles with large-scale thin-layer roughness.

As one can see from Fig. 4, the intensity near backscattering shows a nonsystematic dependence on thin-layer roughening but, at the same time, there appears to be a regular correlation of slopes of the intensities for particles with small-scale and large-scale roughness-the former particles produce more prominent slopes of intensity near $\theta=180^{\circ}$ than the latter particles. The study of polarization near backscattering shows a clear dependence of the depth of the negative polarization branch (NPB) on large-scale thin-layer roughening for icy Gaussian random spheres: roughening decreases the depth of the NPB. The influence of the small-scale thinlayer roughening on the NPB is more complicated. In the case of $x_{\mathrm{cs}}=8$ and 12 roughening decreases the depth of the NPB; whereas, in the case of $x_{\mathrm{cs}}=10$ and 14 , roughening increases the depth. A comparative polarimetric study of both kinds of roughened particles shows that, for $x_{\mathrm{cs}}=8$, the NPB of particles with small-scale roughness coincides with that of particles with large-scale roughness. For the remaining cases, particles with large-scale roughness produce shallower NPBs. 
Maps of intensity, polarization, and the element ratio $D$ of icy particles with thick layers of surface roughness (thickness is 5 dipole diameters) are shown in the fourth and fifth rows of Fig. 3. The fourth row corresponds to particles with smallscale roughness; whereas, the fifth row corresponds to particles with large-scale roughness. It is clear that all maps show noticeable dependencies on surface roughening. For the degree of linear polarization (in the middle), thick-layer surface roughness leads qualitatively to changes similar to those seen in the case of thin-layer surface roughness: polarization increases. The influence of small-scale thick-layer roughening on the intensity is similar to that of thin-layer roughening. However, large-scale roughening changes the intensity map non-monotonically: for $x_{\mathrm{cs}}<12$, it reduces the amplitude of backscattering; whereas, for $x_{\mathrm{cs}}=12$ and 14 , there is an increase of backscattering. $D$ simply decreases for both kinds of thick-layer roughening.

Figure 4 shows also the influence of thick-layer roughening of icy particles on the normalized intensity and polarization near backscattering. Here, the bold shortdashed line corresponds to the case of small-scale roughness; whereas, the bold longdashed line corresponds to large-scale roughness. Recall that the initial particles are presented with the thin solid line. One can see that thick-layer roughening decreases the slope of the intensity curve near $\theta=180^{\circ}$ noticeably. Simultaneously, the NPB is decreased and, for some size parameters (e.g. $x_{\mathrm{cs}}=8$ ), it is washed out completely. Note that some of the results for thick-layer particles with various scales of roughness are similar to those for thin-layer particles; for example, particles with large-scale roughness produce shallower NPBs. At the same time, thick-layer roughening produces shallower NPBs than thin-layer roughening.

Figures 5 and 6 present results for silicate Gaussian random particles $(m=1.6+0.0005 i)$. The figures are organized in the same manner as Figs. 3 and 4. One can see that results for silicate particles confirm most of the findings for icy particles. Thus, we focus on the principal differences from the previous case of icy particles. For the intensity of scattered light they are as follows. Surface roughening of silicate particles does not result in increased backscattering. Particles with large-scale 
roughness produce shallower slopes for the intensity curve near backscattering than particles with small-scale roughness. Contrary to icy particles, thick-layer roughening of silicate particles increases forward scattering.

In the case of polarization, the main feature is the non-monotonic influence of the surface roughening on the NPB. For small values of size parameter $x_{\mathrm{cs}}<10$, roughening results in shallower NPBs; whereas, for $x_{\mathrm{cs}}=10$ and 12 , it enhances the NPBs. Note, especially, that for the latter value of $x_{\mathrm{cs}}$, the NPB increases for all kinds of particles roughness, including thick-layer roughness.

\section{Conclusions}

We have examined scattering of light by roughened and unroughened Gaussian random particles. We have repeated the computations for two refractive indices corresponding to water ice and silicate particles: $m=1.313+0 i$ and $m=1.6+0.0005 i$. For all sets of parameters, scattering of light by roughened particles is noticeably different from scattering by unroughened particles. We find that the most sensitive observable parameter is the degree of linear polarization. Except for the backscattering regime, roughening tends to increase the polarization value. Near backscattering, the dependence is more complicated. In the case of ice particles, roughening tends to decrease the amplitude of the NPB (among the 16 sets of roughened particles there are two exceptions, see Fig. 4). In the case of silicate particles, the influence of surface roughening on the NPB is non-monotonic. For $x_{\mathrm{cs}}<10$, roughening decreases the NPB; whereas, for $x_{\mathrm{cs}}=10$ and 12 it increases NPB. For both refractive indices, particles with large-scale surface roughness produce weaker NPBs than those with small-scale roughness. Finally, particles with thin-layer roughness produce generally more pronounced NPBs than those with thick-layer roughness.

The intensity of scattered light is sensitive to surface roughening mostly near backscattering although, for silicate particles, thick-layer roughening also influences the forward scattering. For relatively large ice particles with thick-layer roughness $\left(x_{\mathrm{cs}}=12\right.$ and 14), we find an amplification of the backscattering intensity; for the remaining cases, roughening decreases backscattering. Particles with thick-layer 
roughness produces slopes in the angular profile of the intensity near $\theta=180^{\circ}$ that are shallower than those produced by unroughened particles or particles with thin-layer roughness.

The influence of surface roughening on the depolarization parameter $D$ depends on the depth of roughening. For shallow roughening, the influence is weak and mainly increases of $D$. However, large-scale roughening of icy sample particles decreases $D$. For deep roughening, $D$ decreases in all cases considered.

Acknowledgments: EZ is grateful to the Academy of Finland for financial support. EZ and YS also thank the CRDF grant UKP2-2614-KH-04. 


\section{References.}

1. B.A. Gustafson and L. Kolokolova, "A systematic study of light scattering by aggregate particles using the microwave analog technique: Angular and wavelength dependence of intensity and polarization.” J. Geophys. Res. 104, 31711-31720 (1999).

2. E.M. Purcell and C.R. Pennypacker, "Scattering and absorption of light by nonspherical dielectric grains.” Astrophys. J. 186, 705-714 (1973).

3. J.J. Goodman, B.T. Draine, and P.J. Flatau, "Application of fast-Fouriertransform techniques to the discrete-dipole approximation." Opt. Lett. 16, 1198-1200 (1991).

4. B.T. Draine and J.J. Goodman, "Beyond Clausius-Mossotti: wave propagation on a polarizable point lattice and the discrete dipole approximation." Astrophys. J. 405, 685-697 (1993).

5. K. Lumme and J. Rahola "Light scattering by porous dust particles in the discrete-dipole approximation.” Astrophys J. 425, 653-667 (1994).

6. E.S. Zubko, M.A. Kreslavskii, and Yu.G. Shkuratov, "The role of scatterers comparable to the wavelength in forming negative polarization of light." Solar Syst. Res. 33, 296-301 (1999).

7. E.S. Zubko, Yu.G. Shkuratov, M. Hart, J. Eversole, G. Videen, "Backscattering and negative polarization of agglomerate particles." Opt. Lett. 28, 1504-1506. (2003)

8. E. Zubko, D. Petrov, Yu. Shkuratov, and G. Videen, "Discrete dipole approximation simulations of scattering by particles with hierarchical structure.” Appl. Opt. 44, 6479-6485 (2005).

9. M.I. Mishchenko, L.D. Travis, D.W. Mackowski "T-matrix computations of light scattering by nonspherical particles: a review.” JQSRT 55, 535-575 (1996).

10. D. Petrov, E. Synelnyk, Yu. Shkuratov and G. Videen "The T-matrix technique for calculations of scattering properties of ensembles of randomly oriented particles with different size.” JQSRT 102, 85-110 (2006). 
11. K.S. Yee, "Numerical solution of initial boundary value problems involving Maxwell's equation in isotropic media." IEEE Trans. Antennas Propag. AP-14, 302-307 (1966).

12. A. Taflove and M.E. Brodwin, "Numerical solution of steady-state electromagnetic scattering problems using the time-dependent Maxwell's equations." IEEE Trans. Microwave Theory Tech, MTT-23, 623-630 (1975).

13. A. Penttilä, E. Zubko, K. Lumme, K Muinonen, M. A. Yurkin, B. Draine, J. Rahola, A. G. Hoekstra, and Yu. Shkuratov "Comparison between discrete dipole implementations and exact techniques." JQSRT (this issue).

14. K. Muinonen, T. Nousianen, P. Fast, K. Lumme, and J.I. Peltoniemi, "Light scattering by Gaussian random particles: ray optics approximation.” JQSRT, 55, 577-601. (1996).

15. C. Li, G.W. Kattawar, P. Yang, "Effects of surface roughness on light scattering by small particles.” JQSRT, 89, 123-131. (2004).

16. W. Sun, T. Nousiainen, K. Muinonen, Q. Fu, N. G. Loeb and G. Videen, "Light scattering by Gaussian particles: A solution with finite-difference time domain technique.” JQSRT, 79-80, 1083-1090 (2003).

17. T. Nousiainen, K. Muinonen, "Surface-roughness effects on single-scattering properties of wavelength-scale particles." JQSRT (this issue).

18. W. Sun, N. Loeb, G. Videen, and Q. Fu, "Examination of surface roughness on light scattering by long ice columns using a 2D FDTD algorithm." Appl. Opt. 43, 1957-1964 (2004).

19.T. Rother, K. Schmidt, J. Wauer, V. Shcherbakov, J.-F. Gayet, "Light scattering on Chebyshev particles of higher order." Appl. Opt. 45, 6030-6037 (2006).

20. M. Born and E. Wolf, Principles of Optics, 7th ed. (Cambridge University Press, Cambridge, 1999).

21.K. Muinonen, E. Zubko, Yu. Shkuratov, J. Tyynelä, and G. Videen "Light scattering by Gaussian random particles with the Discrete-Dipole Approximation". JQSRT (this issue). 


\section{Figure captions.}

Figure 1. Sample Gaussian random spheres.

Figure 2. Sample particles with various kinds of roughness.

Figure 3. Maps of intensity (left), degree of linear polarization (middle), and depolarization parameter $D$ (right) for icy Gaussian random particles $(m=1.313+0 i)$. On each map, a vertical line represents the dependence on the size parameter, whereas the horizontal line represents dependence on the scattering angle. We show results for particles with no surface roughening (first row), with small-scale thin-layer roughness (second row), with large-scale thin-layer roughness (third row), with small-scale thick-layer roughness (fourth row), and with large-scale thick-layer roughness (fifth row).

Figure 4. The normalized intensities and degrees of linear polarization for icy Gaussian random particles $(m=1.313+0 i)$ with differing size parameters $x_{\mathrm{cs}}$. We show results for particles without surface roughening (thin solid line), with small-scale thin-layer roughness (thin line with short dashes), with large-scale thin-layer roughness (thin line with long dashes), with small-scale thick-layer roughness (bold lines with short dashes), and for particles with large-scale thick-layer roughness (bold lines with long dashes).

Figure 5. As in Fig. 3 for silicate particles $(m=1.6+0.0005 i)$.

Figure 6. As in Fig. 4 for silicate particles $(m=1.6+0.0005 i)$. 


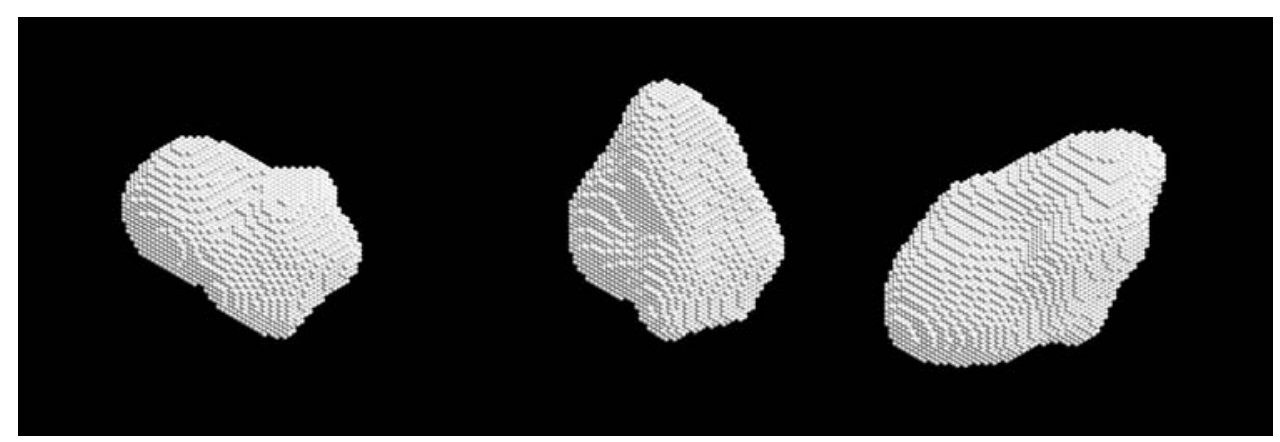

Figure 1. Zubko et al. 


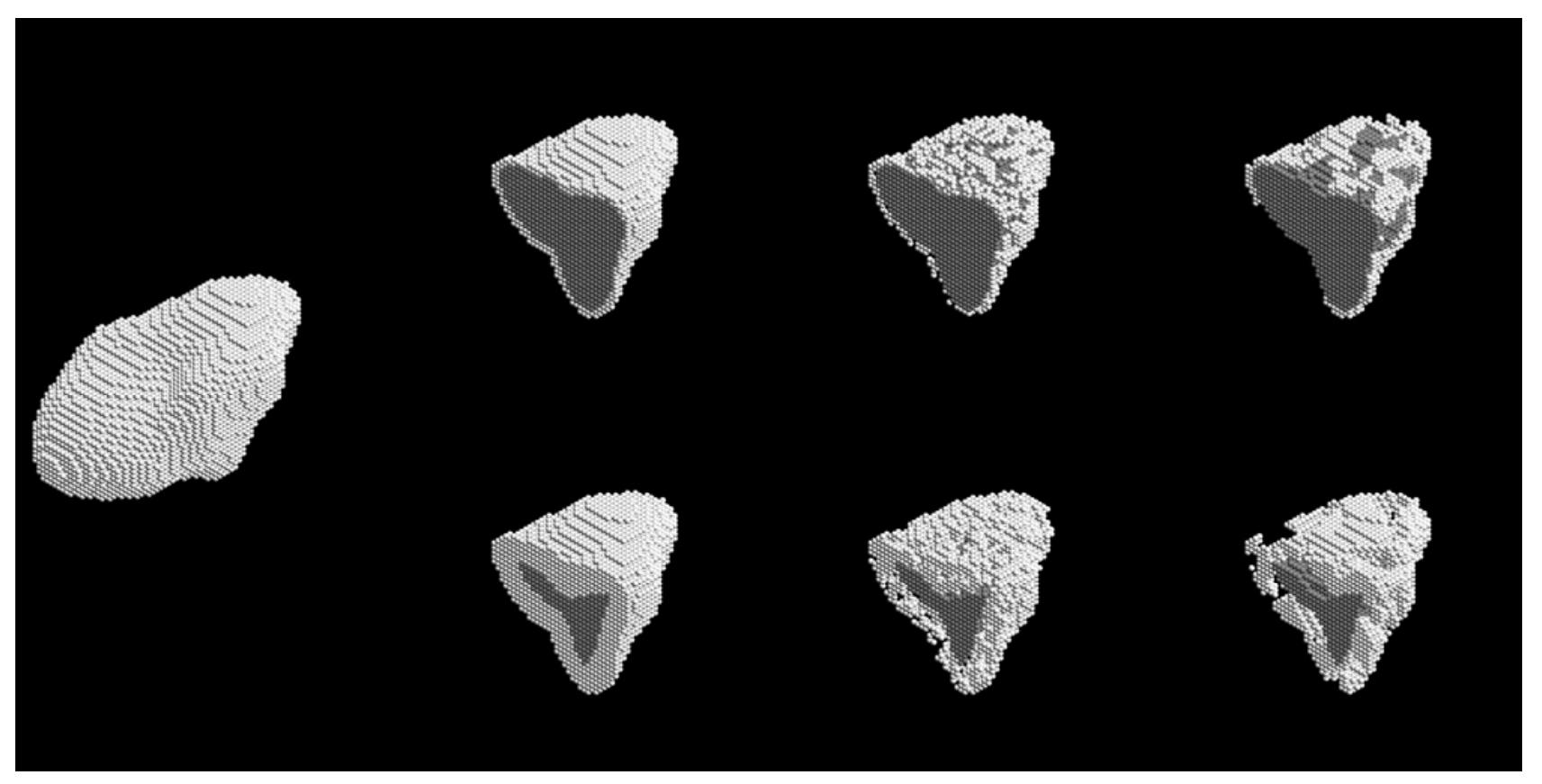

Figure 2. Zubko et al. 

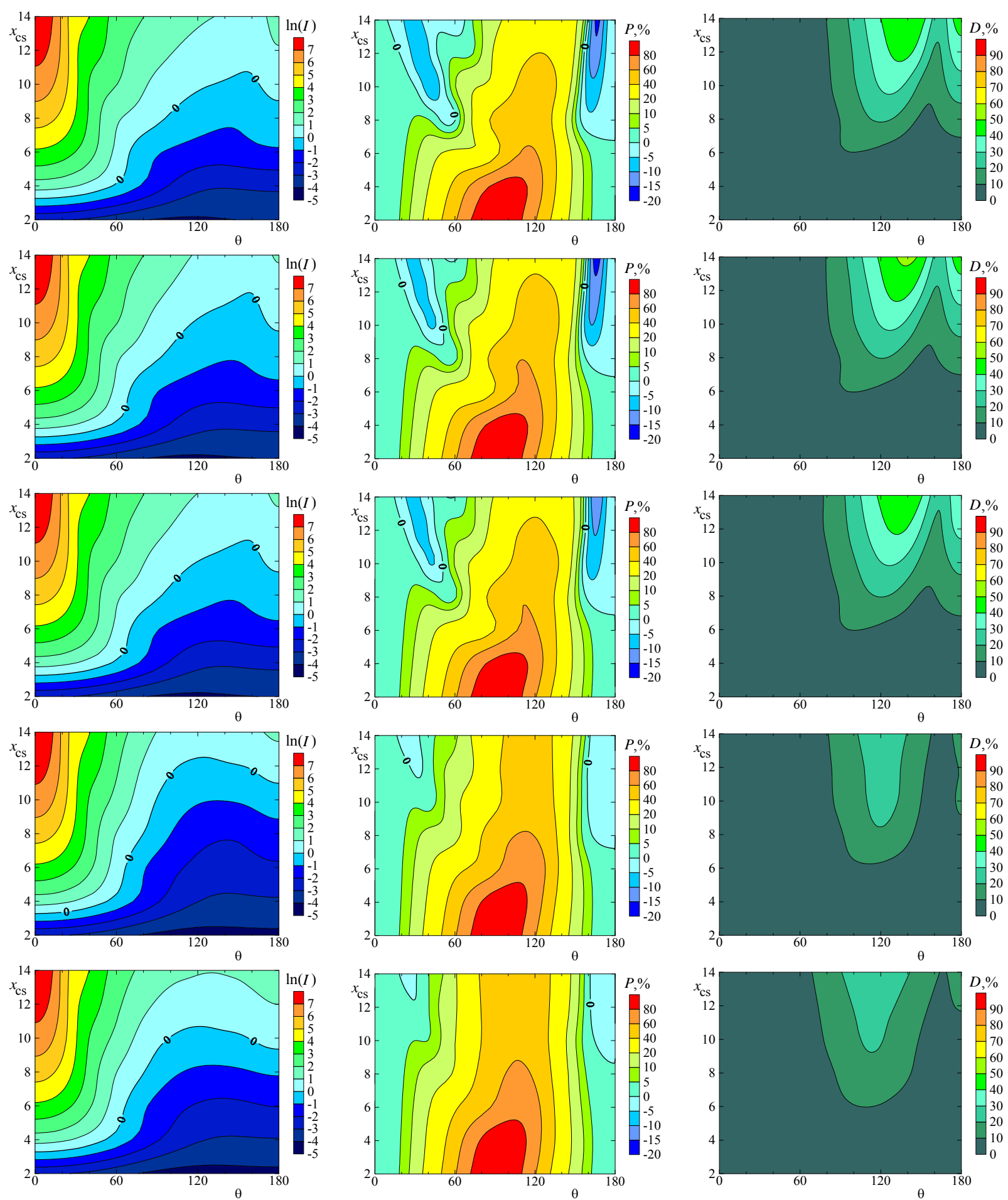

Figure 3. Zubko et al. 


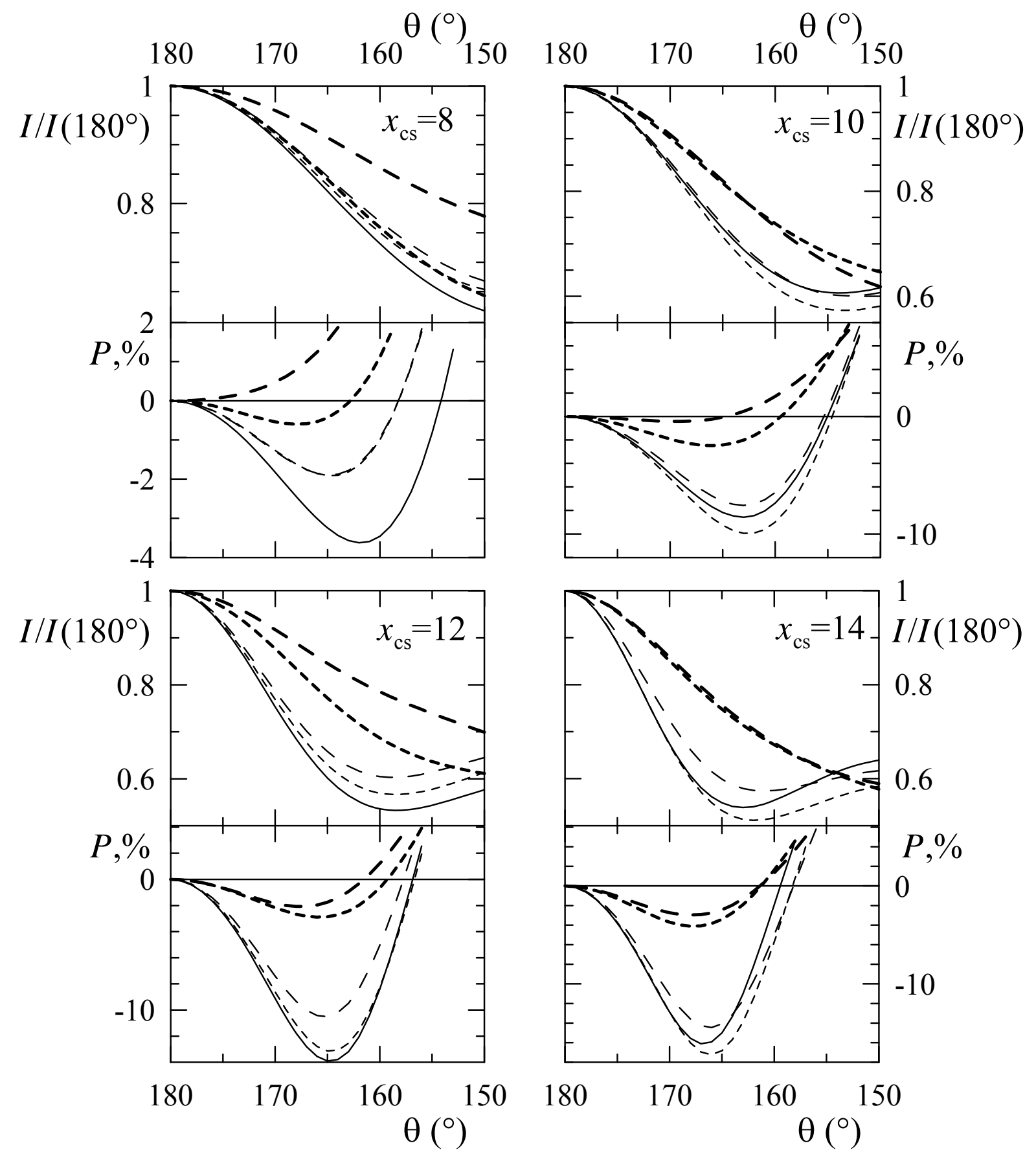

Figure 4. Zubko et al. 

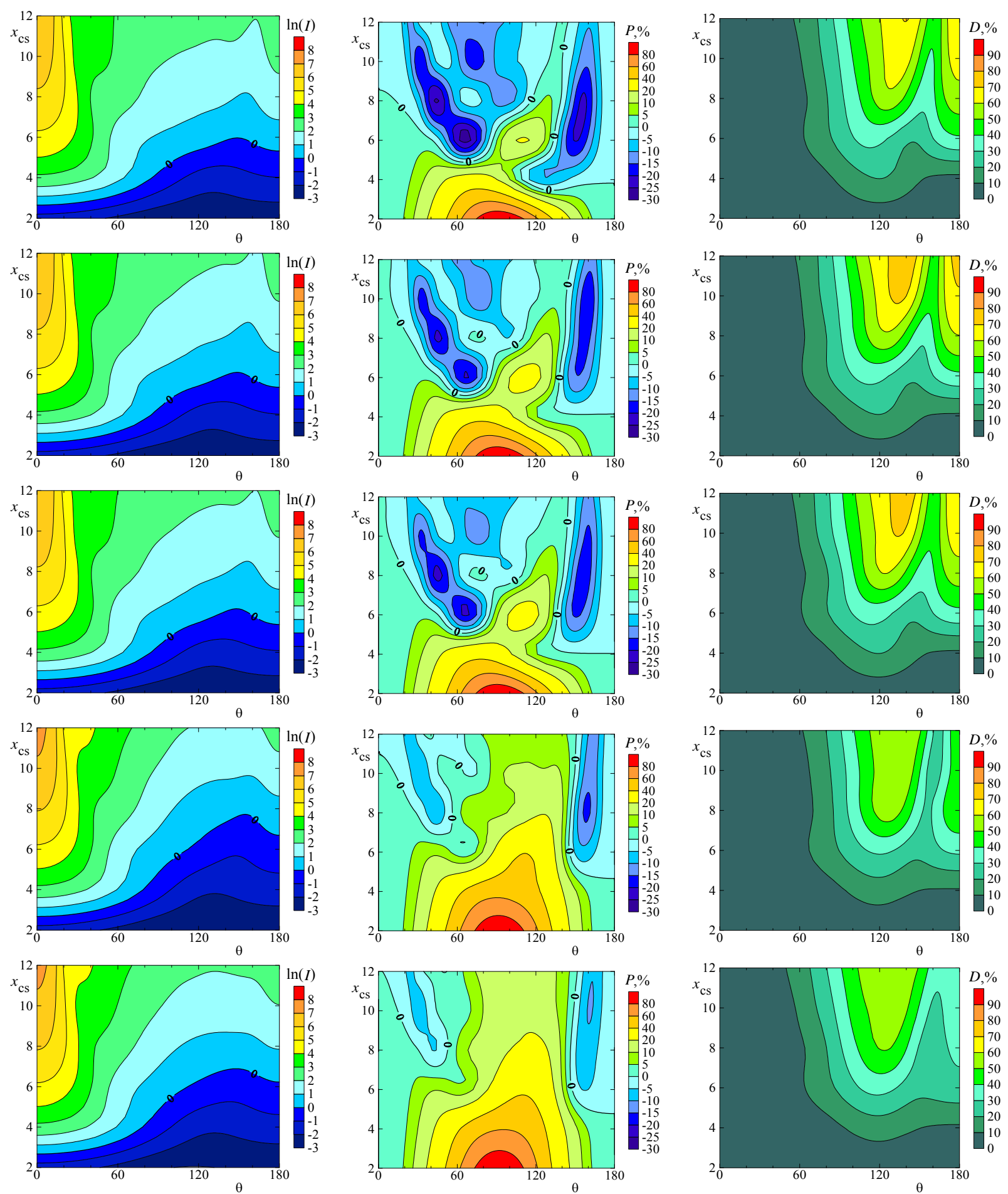

Figure 5. Zubko et al. 


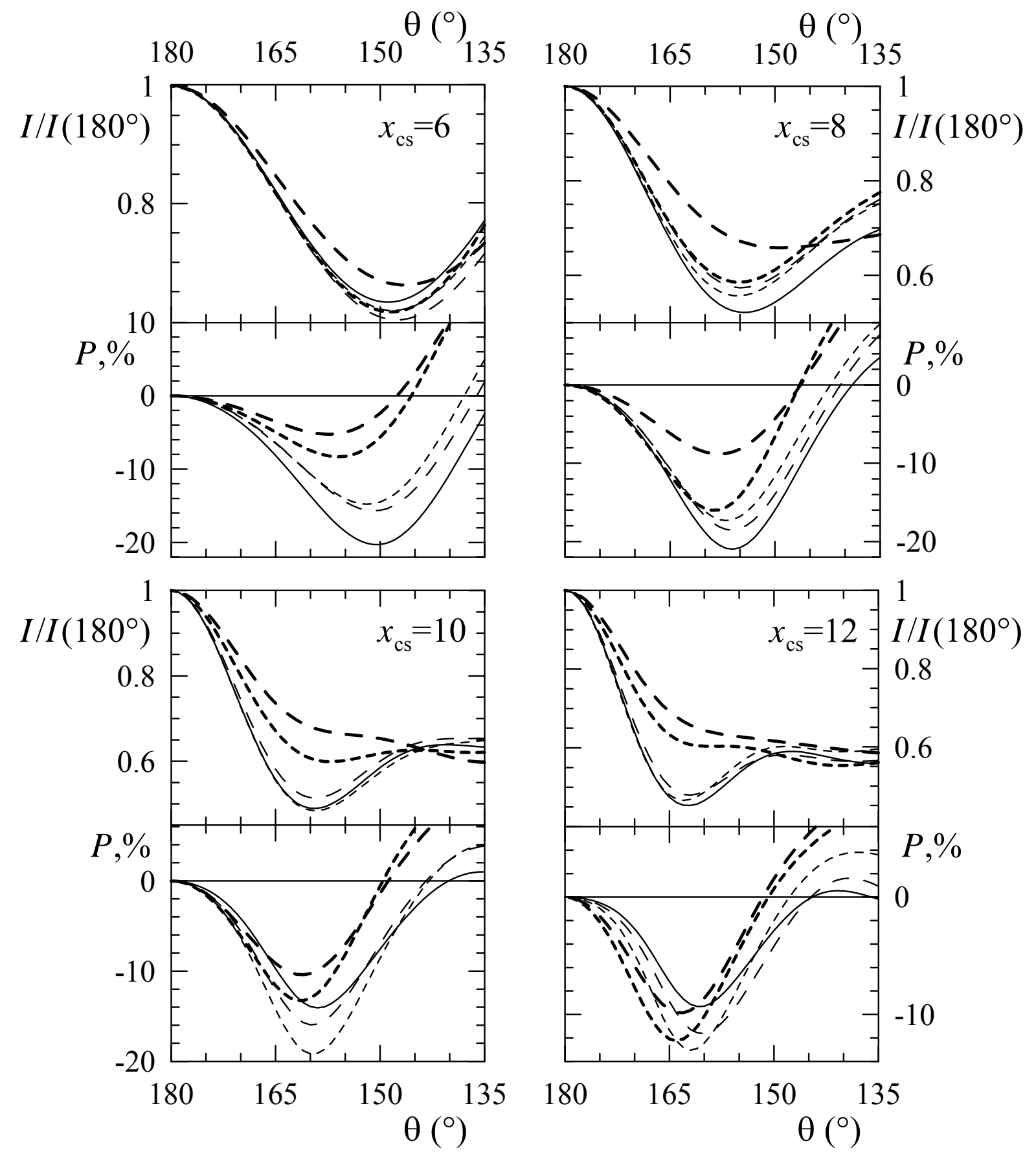

Figure 6. Zubko et al. 